\title{
NOTA
}

\section{AVALIAÇÃO DO REGIME ESTACIONÁRIO EM COLUNAS DE AMOSTRAS DEFORMADAS DE SOLO SOB SATURAÇÃO ${ }^{(1)}$}

\author{
Luciana de Pinho Cunha ${ }^{(2)} \&$ Sérgio Oliveira Moraes ${ }^{(3)}$
}

\begin{abstract}
RESUMO
Estudos sobre o movimento de solutos no solo freqüentemente são realizados em laboratório, sob condições aparentemente controladas. Uma revisão desses estudos mostrou, no entanto, que a variável tempo, para obtenção do regime estacionário tem sido freqüentemente negligenciada. Face ao exposto, o presente trabalho teve por objetivo registrar o tempo para que o fluxo da água no solo sob condições de saturação atinja o equilíbrio dinâmico, medindo-se a condutividade hidráulica saturada $\left(\mathrm{K}_{0}\right)$ do solo. A condição de equilíbrio dinâmico é a etapa que antecede a aplicação do íon no solo em estudos de eluição de solutos. Utilizaram-se amostras deformadas de três solos com diferentes granulometrias: muito argilosa, média e arenosa, coletadas em área experimental da ESALQ/USP. As amostras foram acondicionadas em colunas de PVC, e utilizou-se água destilada e deaerada para saturação e posterior escoamento nas colunas, conforme montagem experimental do permeâmetro de carga constante. Pelos resultados, constatou-se que, nas amostras dos solos muito argiloso e médio, o equilíbrio dinâmico foi atingido após 15 dias de drenagem e, para o arenoso, após 27 dias, sendo a variabilidade de $\mathrm{K}_{0}$ maior nos primeiros seis dias depois de iniciado o ensaio. Essa situação evidenciou que a adoção de um tempo fixo para adição dos solutos pode levar a uma baixa confiabilidade nos resultados.
\end{abstract}

Termos de indexação: condutividade hidráulica, equilíbrio dinâmico, eluição de solutos.

\section{SUMMARY: EVALUATIONOFTHE STATIONARYCONDITIONINDISTURBED SATURATED SOIL COLUMNS}

Studies about solute movement in soil are frequently realized under laboratory conditions, in apparently controlled situations. A review of these studies showed that the variable time, to obtain the stationary condition, is frequently neglected. In view of this fact, the purpose of this study was to evaluate the time it takes until the water flow in saturated soil reaches the steady-state, by measuring saturated hydraulic conductivity

\footnotetext{
(1) Parte da Tese de Mestrado do primeiro autor apresentada à Escola Superior de Agricultura "Luiz de Queiroz" - ESALQ/USP. Recebido para publicação em janeiro de 2006 e aprovado em junho de 2007.

${ }^{(2)}$ Mestre em Agronomia pela Escola Superior de Agricultura "Luiz de Queiroz" - ESALQ/USP. Av. Pádua Dias 11, Caixa Postal 09, CEP 13418-900 Piracicaba (SP). E-mail: cunha_lp@terra.com.br

${ }^{(3)}$ Professor do Departamento de Ciências Exatas, Escola Superior de Agricultura "Luiz de Queiroz" - ESALQ/USP. Av. Pádua Dias 11, Caixa Postal 09, CEP 13418-900 Piracicaba (SP). E-mail: somoraes@esalq.usp.br
} 
$\left(K_{0}\right)$. The steady-state is the stage that precedes the application of the ion into soil samples in solute elution studies. Disturbed soil samples were collected in an experimental area of the ESALQ/USP. The soils had three different granulometries: very clayey, sandy-loam and sandy soil. PVC columns were filled with soil samples and then distilled and deaerated water was used for saturation and subsequent drainage in the columns, according to the experimental set-up of a constant-head permeameter. The data showed that for very clayey and sandy-loam samples, the steady-state was reached after 15 days and for the sandy soil after 27 days. The variability of $K_{0}$ was higher in the first six test days. It was therefore concluded that the use of a fixed time for the addition of solutes in soil can result in a reduction of reliability in results.

Index terms: hydraulic conductivity, steady-state, solute elution.

\section{INTRODUÇÃO}

O regime estacionário, ou "steady-state", caracterizase por um fluxo de água que não varia no tempo e pode ser determinado pela medida da condutividade hidráulica saturada $\left(\mathrm{K}_{0}\right)$. A condutividade hidráulica saturada é um característica de elevada importância para estudar o fluxo de água e de íons no solo, pois indica como uma solução aquosa se move no meio poroso. Em solo saturado e de estrutura estável, $\mathrm{K}_{0}$ é constante e sua ordem de grandeza é de $10^{-4}$ a $10^{-5}$ $\mathrm{ms}^{-1}$ em materiais arenosos e de $10^{-6}$ a $10^{-9} \mathrm{~ms}^{-1} \mathrm{em}$ materiais argilosos (Hillel, 1980). No entanto, até que seja atingido $\mathrm{um}$ valor constante, $\mathrm{K}_{0}$ sofre alterações, pois é dependente das propriedades do meio poroso e do líquido permeante (Radcliffe \& Rasmussen, 2000), já que diferentes tipos de água podem ser utilizados para estabelecer a condutividade hidráulica saturada de uma amostra.

A escolha do método utilizado neste trabalho foi baseada no fato de que estudos de deslocamento e propriedades de movimento de solutos utilizam, na maioria das vezes, amostras de solo deformado, saturada com fluxo constante unidimensional (direção - z), coletando o efluente durante o tempo de infiltração (Corrêa et al., 1999; Matos et al., 1999; Lee et al., 2000; Paseka et al., 2000; Martinez et al., 2001; Azevedo et al., 2003; Shukla et al., 2003).

Estudos para obtenção de medidas de movimento de solutos são baseados na teoria de deslocamento miscível, a qual considera que um fluido que contém um íon a ser estudado é deslocado pelo mesmo fluido sem o íon, através de um volume de solo (Nielsen \& Biggar, 1961). Para essa estimativa são utilizados programas numéricos, como CFITIM ou CXTFIT, que ajustam soluções analíticas da equação de transporte a curvas experimentais de eluição, chamadas de "breakthrough curve" (Inoue et al., 2000), dadas pela concentração relativa $\mathrm{C} / \mathrm{C}_{0}$ (em que $\mathrm{C}$ é a concentração do efluente coletado e $\mathrm{C}_{0}$ a concentração inicial da solução que contém o íon a ser estudado), em função do tempo acumulado ou do volume de efluente coletado (em unidade de volume de poro, que corresponde ao volume de fluido que cabe na coluna de solo).
No entanto, durante a lavagem das colunas observou-se que o número de horas para dar início ao ensaio de eluição parecia ser um tempo arbitrário, que não necessariamente coincidia com a condição de fluxo sob equilíbrio dinâmico, ou seja, o fluxo não havia atingido o "steady-state". Portanto, o objetivo deste trabalho foi avaliar o tempo necessário para atingir o "steady-state", por meio das medidas de condutividade hidráulica saturada em laboratório - etapa experimental nos estudos de eluição, que antecede a aplicação do soluto de interesse.

\section{MATERIAL E MÉTODOS}

Os ensaios foram realizados no Laboratório de Física do Solo do Departamento de Ciências Exatas da Escola Superior de Agricultura "Luiz de Queiroz" ESALQ/USP, situada no município de Piracicaba-SP. As unidades experimentais consistiram de colunas de PVC preenchidas com as amostras dos solos, submetidas à saturação e posterior drenagem sob carga constante.

As amostras foram coletadas em área experimental da ESALQ/USP, a uma profundidade de $0-20 \mathrm{~cm}$, e a escolha da área teve por objetivo utilizar solos com alto, médio e baixo teor de argila. As amostras pertencem aos solos classificados como Terra Roxa Estruturada, Latossolo Vermelho-Escuro e Latossolo Vermelho-Amarelo, denominados solo 1, 2 e 3, respectivamente. Os dados de granulometria, densidade do solo dentro das colunas depois de acondicionado e respectivo coeficiente de variação, que permite avaliação da precisão dos dados, encontram -se no quadro 1.

As amostras dos três solos tiveram o mesmo preparo antes de serem levadas ao laboratório. Após secagem ao ar, procedeu-se ao destorroamento e peneiramento em peneira de malha de $2 \mathrm{~mm}$.

As colunas tinham $23 \mathrm{~cm}$ de altura e $5 \mathrm{~cm}$ de diâmetro interno e foram preenchidas com terra até $20 \mathrm{~cm}$ de altura, sendo três colunas para cada amostra de solo. Cada coluna foi saturada lentamente, no sentido vertical ascendente, com água destilada e deaerada, para que o ar presente na coluna fosse expulso ou dissolvido (Moraes, 1991). A montagem 
Quadro 1. Valores de granulometria, densidade do solo dentro das colunas e respectivo coeficiente de variação

\begin{tabular}{|c|c|c|c|c|c|}
\hline \multirow{2}{*}{ Tipo de solo } & \multicolumn{3}{|c|}{ Granulometria } & \multirow{2}{*}{ Densidade do solo dentro da coluna } & \multirow{2}{*}{$\mathrm{CV}$} \\
\hline & Areia & Silte & Argila & & \\
\hline & \multicolumn{3}{|c|}{$\mathrm{g} \mathrm{kg}^{-1}$} & $\mathrm{~kg} \mathrm{~m}^{-3}$ & $\%$ \\
\hline Solo 1 & 204 & 172 & 624 & 1,32 & 0,41 \\
\hline Solo 2 & 371 & 119 & 461 & 1,39 & 0,52 \\
\hline solo 3 & 706 & 115 & 178 & 1,65 & 0,27 \\
\hline
\end{tabular}

experimental utilizada foi o permeâmetro de carga constante (Youngs, 1991), mantendo-se uma carga hidráulica de $2 \mathrm{~cm}$.

A água foi coletada em recipientes com capacidade de $23 \mathrm{~mL}$, para quantificar o volume de água em cada frasco. Conhecendo o volume e o tempo necessário para atingi-lo, foi possível medir a vazão e, assim, obter a condutividade hidráulica saturada de cada solo pela equação de Darcy (Libardi, 2005).

O tempo inicial $\left(\mathrm{t}_{0}\right)$ coincidiu com o momento em que a coluna era fixada em um suporte e conectada ao frasco de Mariotte e o fluxo descendente se iniciava. O tempo foi monitorado por cronômetro de precisão de $1 / 100 \mathrm{~s}$, e a temperatura local, por termômetro, de forma que foi possível observar que a temperatura se manteve em torno de $25^{\circ} \mathrm{C}$, com variação de $\pm 3{ }^{\circ} \mathrm{C}$ ao longo do experimento, porém não ultrapassando $1{ }^{\circ} \mathrm{C}$ durante um dia de medida.

A primeira medida $\left(t_{1}\right)$ foi realizada entre 1 e $2 \mathrm{~h}$ após $\mathrm{t}_{0}$, e a partir daí foi coletada uma bateria de dados, correspondente ao número de frascos coletados durante o dia. No tempo $t_{2}$, cerca de 24 h após $t_{1}$, foi coletada nova bateria, no tempo $\mathrm{t}_{3}$, em torno de $24 \mathrm{~h}$ após o tempo $t_{2}$, mais uma bateria, e assim por diante, até o término do ensaio, quando a condutividade hidráulica saturada era constante.

Portanto, cada frasco foi considerado uma repetição da condutividade hidráulica saturada, ou seja, com base em todos os frascos coletados no mesmo tempo $t_{i}$, para cada três colunas da mesma amostra de solo, foram calculadas as médias de condutividade e os respectivos desvios-padrão e coeficientes de variação.

\section{RESULTADOS E DISCUSSÃO}

\section{Amostra do solo 1}

A variação de $K_{0}$ para a amostra do solo 1 pode ser visualizada na figura 1 , que apresenta as curvas de condutividade hidráulica saturada em função do tempo, das três colunas do solo 1, e a média das repetições, com os desvios-padrão.

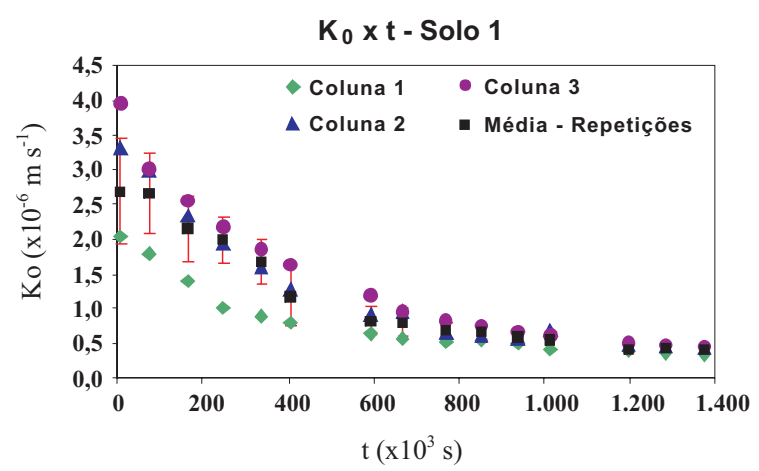

Figura 1. Condutividade hidráulica saturada em função do tempo (solo 1), média das repetições e respectivos desvios-padrão (barras verticais).

De acordo com os dados obtidos, o fluxo decresceu acentuadamente até o sétimo ponto da curva, $t_{7}$ $\left(594,54 \times 10^{3} \mathrm{~s}\right)$, que corresponde aproximadamente aos primeiros sete dias de ensaio. A partir de então, passou a apresentar tendência a atingir o equilíbrio dinâmico, o que só ocorreu aproximadamente entre 13 e 15 dias $\left(1.198,62\right.$ e $\left.1.373,04 \times 10^{3} \mathrm{~s}\right)$ depois do início da lavagem das colunas.

$\mathrm{O}$ valor final de $\mathrm{K}_{0}$ atingido foi de $0,4127 \mathrm{~m} \mathrm{~s}^{-1}$ para o solo 1; após ter sido observado que o fluxo já havia atingido o "steady-state", ele foi de aproximadamente $15 \%$ do valor inicial, $0,7110 \times 10^{-6} \mathrm{~m} \mathrm{~s}^{-1}$, ou seja, este solo sofreu decréscimo de $85 \%$ no valor de $\mathrm{K}_{0}$. Ao considerar, portanto, que o "steady-state" ocorreu em torno de 15 a 16 dias após início da lavagem das colunas, esse seria o tempo mais indicado para início do ensaio de eluição, no caso da amostra deste solo.

\section{Amostra do solo 2}

O solo 2 apresentou diminuição mais rápida e acentuada de $\mathrm{K}_{0}$ nos cinco primeiros tempos medidos (Figura 2), em que $t_{5}\left(347,58 \times 10^{3} \mathrm{~s}\right)$ corresponde a aproximadamente quatro dias após o início do ensaio e que passou a apresentar tendência ao "steady-state" nos três últimos tempos da curva. Essa figura mostra que a coluna 1 não possui medições nos dois últimos tempos, e isso ocorreu devido ao fato de que, após 
aproximadamente 12 dias de ensaio, a coluna 1 encontrava-se totalmente drenada, mesmo estando ainda ligada ao frasco de Mariotte e este contendo água. Atribuiu-se esse comportamento a prováveis mudanças de temperatura no laboratório, uma vez que a condutividade hidráulica varia aproximadamente $3 \%$ para cada $1{ }^{\circ} \mathrm{C}$ de mudança na temperatura (Youngs, 1991; Daniel, 1994). Embora a amplitude de variação possa ser considerada pequena ao longo do experimento, ela pode ter influenciado o sistema, fazendo com que o fornecimento de água fosse encerrado, permitindo dessa forma que a coluna secasse. Essa mudança ocorreu no final do experimento, quando a quantidade de água no frasco de Mariotte era menor e o valor de $\mathrm{K}_{0}$ também era reduzido, ficando mais sujeitos a alterações conforme a temperatura; o experimento mostrou, portanto, maior sensibilidade ao final do ensaio. Pôde-se notar que esse efeito é atenuado se o frasco de Mariotte for mantido cheio.

$\mathrm{O}$ valor final de $\mathrm{K}_{0}$ encontrado, $0,2245 \times 10^{-6} \mathrm{~m} \mathrm{~s}^{-1}$, corresponde a aproximadamente $32 \%$ do valor inicial, $0,7110 \times 10^{-6} \mathrm{~m} \mathrm{~s}^{-1}$, para este solo, ou seja, a amostra do solo 2 apresentou queda de $68 \%$ em relação ao seu valor inicial. O equilíbrio foi atingido aproximadamente após 15 dias - tempo sugerido para início do ensaio de eluição neste solo.

\section{Amostra do solo 3}

A exemplo dos outros solos, observou-se queda bastante acentuada de $\mathrm{K}_{0}$ até o sexto tempo (que corresponde aproximadamente aos sete primeiros dias de ensaio) (Figura 3). A partir do sexto tempo a condutividade hidráulica saturada passou a apresentar diminuição menor e lenta, até o solo atingir o equilíbrio dinâmico.

A condutividade hidráulica saturada final, $0,3253 \mathrm{~m} \mathrm{~s}^{-1}$, teve valor correspondente a $18 \%$ do valor inicial, 1,7741 $\mathrm{m} \mathrm{s}^{-1}$, ou seja, $\mathrm{K}_{0}$ teve redução de

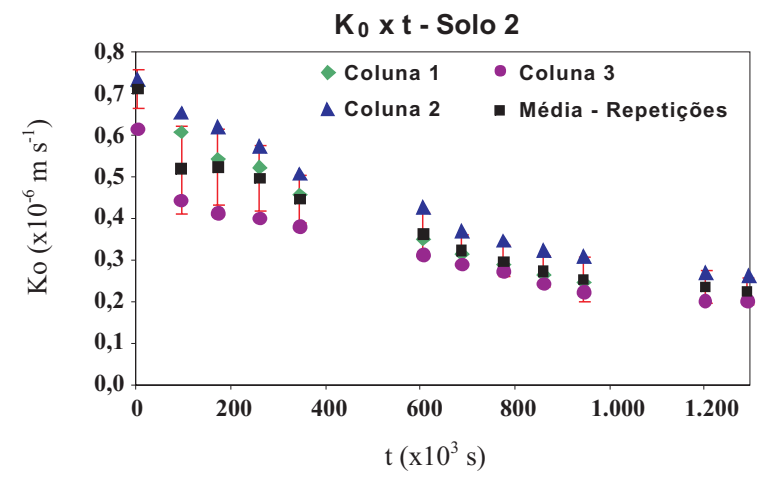

Figura 2. Condutividade hidráulica saturada em função do tempo (solo 2), média das repetições e respectivos desvios-padrão (barras verticais).
$82 \%$ para este solo. A amostra do solo 3 levou aproximadamente 27 dias para atingir o equilíbrio dinâmico - tempo aconselhável para o início do ensaio de eluição neste solo.

\section{Comparação dos dados das três amostras de solo estudadas}

As amostras dos solos 2 e 3, mesmo possuindo teor menor de argila, apresentaram valores de média inicial e final menores que as do solo 1 , o que, de acordo com Reichardt \& Timm (2004), deveria influenciar para que houvesse $\mathrm{K}_{0}$ menor no solo 1 , ou seja, um solo mais arenoso, em geral, deveria ter condutividade hidráulica saturada maior que a de um solo argiloso (Figura 4).

Essa discrepância talvez possa ser explicada pelo fato de as amostras de solo serem deformadas. O processo de preparação destrói os macroporos no solo, fazendo com que a influência destes, segundo Starrett et al. (1996), seja eliminada. Dessa forma, as amostras apresentariam estrutura similar dentro das colunas, o que explicaria o fato de os valores de $\mathrm{K}_{0}$ finais serem muito próximos entre si, sendo o maior valor de $\mathrm{K}_{0}$ final (solo 1 ) apenas 1,84 vez o menor valor (solo 2).

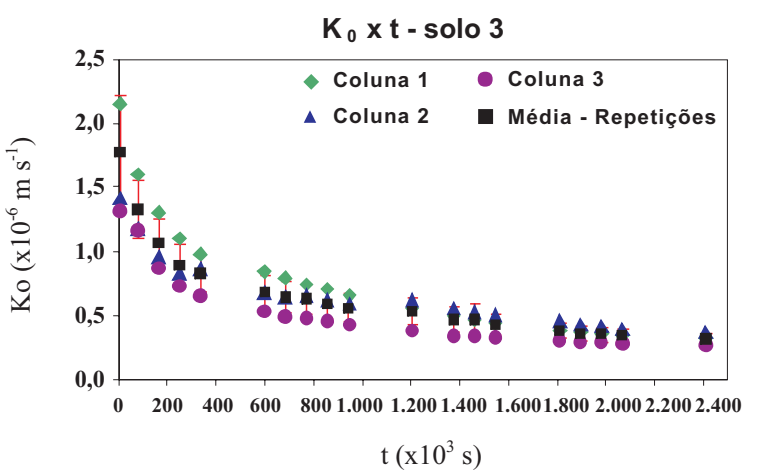

Figura 3. Condutividade hidráulica saturada em função do tempo (solo 3), média das repetições e respectivos desvios-padrão (barras verticais).

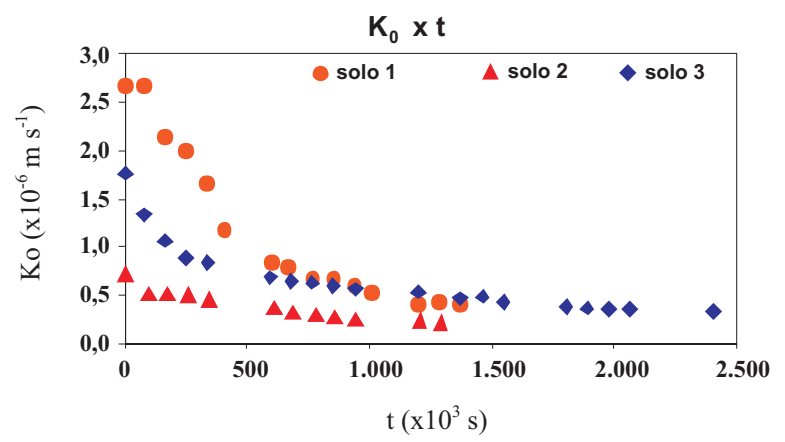

Figura 4. Média das condutividades hidráulicas saturadas, em função do tempo, para os três solos. 
O efeito dos poros grandes, de acordo com Mesquita \& Moraes (2004), permite que um solo argiloso tenha valores de $\mathrm{K}_{0}$ similares aos de solos arenosos, contribuindo esses poros, portanto, para maior condutividade hidráulica saturada. Dessa forma, é possível concluir, de acordo com os dados obtidos, que, no caso de amostras indeformadas, por serem peneiradas, acondicionadas e compactadas, a ausência desses poros grandes em solos mais arenosos poderia diminuir a condutividade hidráulica saturada deles, fazendo com que se comportem como solos argilosos.

As três amostras de solo apresentaram valores de $\mathrm{K}_{0}$ entre $10^{-6}$ e $10^{-7} \mathrm{~m} \mathrm{~s}^{-1}$. O solo 1 variou de $2,5546 \mathrm{x}$ $10^{-6}$ a $3,9428 \times 10^{-7} \mathrm{~m} \mathrm{~s}^{-1}$; o solo 2 , de $7,1103 \times 10^{-7} \mathrm{a}$ $2,2453 \times 10^{-7} \mathrm{~m} \mathrm{~s}^{-1}$; e o solo 3 , de $1,7741 \times 10^{-6}$ a $3,2525 \times 10^{-7} \mathrm{~m} \mathrm{~s}^{-1}$. No entanto, é possível observar que, nos três solos, a redução mais acentuada em $\mathrm{K}_{0}$ ocorreu até o quinto e sexto dias do ensaio, apresentando a partir desse ponto tendência ao equilíbrio dinâmico, passando a condutividade a apresentar diminuição menor até atingir o "steadystate".

Atribui-se essa queda rápida e acentuada de $\mathrm{K}_{0}$ no início do ensaio e posteriormente menor a dois efeitos principais: à dispersão das partículas na amostra, ou seja, à separação e migração destas para o interior dos poros, e à interação da água com a matriz do solo. Esses efeitos são provavelmente mais intensos no início da lavagem das colunas; após certo tempo, o líquido percolante e as partículas do solo tendem ao equilíbrio, o que faz com que a queda de $\mathrm{K}_{0}$ se torne mais discreta, até que seja atingido o "steady-state".

A dispersão ocorreu provavelmente devido à diminuição das forças de atração entre as partículas de argila. A força é inversamente proporcional à constante dielétrica do meio e diminui com a entrada da água, uma vez que esta possui maior constante dielétrica que o ar nos poros do solo - aproximadamente 1 para o ar e 80 para a água -, provocando assim a separação das partículas.

Quando se inicia o fluxo descendente, há dispersão dessas partículas, o que, de acordo com Andrade \& Cruciani (1996), causa diminuição ou fechamento dos poros da amostra. Dessa forma, a condutividade hidráulica saturada apresenta decréscimo considerável até que haja rearranjo das partículas no interior da amostra, já que, pela lei de Poiseuille, a vazão é diretamente proporcional à quarta potência do raio do poro.

A dispersão de partícula foi evidenciada pela presença de resíduos de argila no efluente de todas as colunas a partir do segundo dia de ensaio, para os três solos, resultado do arraste de partículas finas das amostras, indicando sua dispersão e migração, como discutido por Barreto et al. (2001). No entanto, passados dois dias, a água que fluía da coluna encontrava-se limpa novamente, levando à idéia de provável equilíbrio entre as partículas dentro da amostra.
Já a interação entre as partículas do solo e o líquido percolante pode propiciar menor valor de $\mathrm{K}_{0}$ de duas formas: pela proximidade da água em relação à superfície da argila, como explicado por Iwata et al. (1995), o que pode aumentar a viscosidade da água nessa região, diminuindo o seu movimento e, portanto, o $\mathrm{K}_{0}$, e pela espessura da dupla camada difusa, que causa estreitamento nos poros condutores.

Uma evidência da influência da dupla camada difusa pode ser o fato de a diminuição de $\mathrm{K}_{0}$ ter sido muito maior na amostra do solo mais argiloso (solo 3), mesmo tendo este solo apresentado valores iniciais e finais maiores que os das amostras dos solos 1 e 2 . Isto ocorreu provavelmente pelo fato de as partículas de argila serem negativamente carregadas, tendo ligado a elas maior número de íons; assim, este solo foi mais afetado pela entrada da água destilada. A água se mistura aos íons ligados às partículas de argila, diminuindo a concentração iônica nos poros do solo, o que provoca aumento na espessura da dupla camada difusa (Pupisky \& Shainberg, 1979; Mitchell, 1993). Uma vez que a dupla camada sofre alterações devido à entrada da água nos poros do solo, assim que a solução atinge concentração de equilíbrio no interior destes, $\mathrm{K}_{0}$ passa a ter redução menor, até atingir o "steady-state".

A amostra do solo 1 levou aproximadamente 15 dias para atingir o "steady-state", assim como a amostra do solo 2, o que está de acordo com o que foi apresentado por Azevedo et al. (2003), enquanto para a amostra do solo 3 foi necessário um período de 27 dias para que se constatasse o equilíbrio dinâmico; portanto, não seria aconselhável tomar como base um tempo único para todos os solos em estudo.

Os dados finais da condutividade hidráulica saturada no tempo, encontrados por Azevedo et al. (2003) num ensaio que durou aproximadamente 17 dias, foram entre $1,2 \times 10^{-8}$ e $1,8 \times 10^{-8} \mathrm{~m} \mathrm{~s}^{-1}$, tendendo os valores ao equilíbrio a partir do sexto dia de ensaio, em conformidade com o que foi obtido neste trabalho. Nesse caso, os autores associaram tal decréscimo à lixiviação de sais solúveis no solo, devido ao uso de água destilada, que, como explicado anteriormente, diminui a concentração eletrolítica do fluido nos poros, causando expansão da dupla camada difusa. Como o objetivo desses autores era realizar ensaios de eluição, eles chamam a atenção para o fato de que o valor inicial de $\mathrm{K}_{0}$ com a solução era o final de $\mathrm{K}_{0}$ com água destilada, como é sugerido no presente trabalho.

A diminuição da condutividade hidráulica saturada percolada com água destilada, observada neste trabalho, foi também constatada por Levy et al. (2005). Estudando solos semi-áridos de Israel, eles combinaram efeitos de salinidade da água e associaram a diminuição de $\mathrm{K}_{0}$ à expansão da argila, já que não encontraram argila dispersa no líquido percolado. No entanto, no presente trabalho, como foi 
observado material no efluente, atribuiu-se também essa diminuição à migração das partículas de argila devido à diminuição das forças de atração entre elas.

Outros autores que encontraram diminuição considerável na condutividade foram Paseka et al. (2000), que observaram redução de quase metade da velocidade de infiltração em colunas de solo com amostras deformadas, associando esse efeito às reações de trocas de cátion e, ainda, à expansão dos minerais de argila. Esses autores concluíram que fatores que causam variações temporais em laboratório deveriam ser mais investigados em estudos de modelos de transporte.

$\mathrm{O}$ efeito da diminuição de $\mathrm{K}_{0}$ no tempo, portanto, pode ser resultado da interação entre a água destilada e a matriz do solo, bem como da dispersão e migração das partículas coloidais. O primeiro se deve ao fato de a água destilada apresentar concentração iônica muito baixa $\left(1,3 \times 10^{-5} \mathrm{~mol} \mathrm{~L}^{-1}\right)$ quando comparada com a de outras soluções, como, por exemplo, a solução-padrão de $\mathrm{CaSO}_{4} 0,02 \mathrm{~mol} \mathrm{~L}^{-1}$; o segundo efeito se deve à diminuição da força de atração entre as partículas, por causa da entrada da água nos poros do solo, diminuindo assim o espaço poroso disponível para o fluxo, como observado por Keren \& Singer (1988).

No final do ensaio, quando as colunas foram desmontadas, notou-se que os três primeiros centímetros, aproximadamente, da parte superior encontravam-se mais úmidos que a parte inferior. Era possível perceber que a textura na parte superior era pastosa e, na parte inferior, apesar da saturação, era mais firme; provavelmente, devido à migração das partículas, a camada inferior sofreu adensamento, diminuindo o fluxo de água e fazendo com que esta se concentrasse mais na faixa superior da coluna, ou seja, é possível que a condutividade hidráulica saturada não tenha se mantido constante ao longo da coluna.

Pelas figuras, é possível observar ainda maior variabilidade das amostras em torno da média no início dos ensaios, mais precisamente nos seis primeiros dias, indicando que maior número de amostras é necessário para se obter maior confiança nessa etapa do experimento. Mesquita et al. (2003), considerando os altos coeficientes de variação para a variabilidade espacial, afirmam que $\mathrm{K}_{0}$ requer grande número de análises para manter a mesma confiabilidade e permitir comparações.

Considerando que o interesse seria obter as curvas de eluição de um soluto, se os ensaios fossem iniciados após um dia, visto que os tempos encontrados foram de 15 e 27 dias, depois do início da lavagem das colunas, ter-se-ia maior variabilidade e, portanto, menor confiabilidade nos dados em relação ao ensaio iniciado após ser constatado o "steady-state". Ou seja, para se obter resposta mais confiável dos dados nos estudos de movimento de íons, é necessário que a condutividade hidráulica saturada do solo com água tenha atingido o "steady-state".

\section{CONCLUSÕES}

1. Em razão das diferenças no tempo para atingir o equilíbrio dinâmico - 15 dias para a amostra do solo 1,15 dias para a do solo 2 e 27 dias para a do solo 3 -, não se deve adotar para amostras de solos distintos o mesmo tempo para o início do ensaio de eluição.

2. A condutividade hidráulica saturada apresentou maior variabilidade no início dos ensaios, mais propriamente nos seis primeiros dias, o que pode levar a uma menor confiabilidade nos experimentos de movimento de solutos.

\section{LITERATURA CITADA}

ANDRADE, L.N.V. \& CRUCIANI, D.E. Condutividade hidráulica no processo de eluição em um solo Bruno-nãocálcico. Sci. Agríc., 53:43-50, 1996.

AZEVEDO, I.C.D.; NASCENTES, C.R.; AZEVEDO, R.F.; MATOS, A.T. \& GUIMARÃES, L.M. Coeficiente de dispersão hidrodinâmica e fator de retardamento de metais pesados em solo residual compactado. Solos Rochas, 26:229-249, 2003.

BARRETO, A.N.; OLIVEIRA, G.R.; NOGUEIRA, L.C. \& IVO, W.M.P.M. Condutividade hidráulica saturada em um solo aluvial do perímetro irrigado de São Gonçalo, PB. R. Bras. Eng. Agric. Amb., 5:152-155, 2001.

CORREAA, M.M.; LIMA, L.A.; MARTINEZ, M.A.; RIGITANO, R.L.O. \& SAMPAIO, S.C. Deslocamento miscível de sulfona de aldicarbe em colunas de solo. R. Bras. Eng. Agric. Amb., 3:217-221, 1999.

DANIEL, D.E. State-of-the-art: Laboratory hydraulic conductivity tests for saturated soils. In: DANIEL, D.E. \& TRAUTWEIN, S.J., eds. Hidraulic conductivity and waste contaminant transport in soil. Philadelphia, ASTM, 1994. p.30-78.

HILLEL, D. Fundamentals of soil physics. New York, Academic Press, 1980. 413p.

INOUE, M.; SIMUNEK, J.; SHIOZAWA, S. \& HOPMANS, J.W. Simultaneous estimation of soil hydraulic and solute transport parameters from transient infiltration experiments. Adv. Water Res.,23:677-688, 2000.

IWATA, S.; TABUCHI, T. \& WARKENTIN, B.P. Soil-water interactions: Mechanisms and applications. 2.ed. New York, M. Dekker, 1995. 440p.

KEREN, R. \& SINGER, M.J. Effect of low electrolyte concentration on hydraulic conductivity of sodium/ calcium-montmorillonite-sand system. Soil Sci. Soc. Am. J., 52:368-373, 1988.

LEE, J.; JAYNES, D.B. \& HORTON, R. Evaluation of a simple method for estimating solute transport parameters: Laboratory studies. Soil Sci. Soc. Am. J., 64:492-498, 2000. 
LEVY, G.J.; GOLDSTEIN, D. \& MAMEDOV, A.I. Saturated hydraulic conductivity of semiarid soils: Combined effects of salinity, sodicity, and rate of wetting. Soil Sci. Soc. Am. J., 69:653-662, 2005.

LIBARDI, P.L. Dinâmica da água no solo. São Paulo, Edusp, 2005. 335p.

MARTINEZ, M.A.; RAMOS, V.B.N.; OLIVEIRA, R.A. \& COSTA, S.N. Influência da competição catiônica nos valores de fator de retardamento e coeficiente de dispersão-difusão de zinco e cobre no solo. R. Bras. Eng. Agric. Amb., 5:211$215,2001$.

MATOS, A.T.; COSTA, L.M.; FONTES, M.P.F. \& MARTINEZ, M.A. Retardation factors and dispersion-diffusion coefficients of $\mathrm{Zn}, \mathrm{Cd}, \mathrm{Cu}$, and $\mathrm{Pb}$ in soils from ViçosaMG, Brazil. Trans. ASAE, 42:903-910, 1999.

MESQUITA, M.G.B.F. \& MORAES, S.O. A dependência entre a condutividade hidráulica saturada e atributos físicos do solo. Ci. Rural, 34:963-969, 2004.

MESQUITA, M.G.B.F.; MORAES, S.O. \& CORRENTE, J.E. Caracterização estatística de variáveis físicas do solo. Acta Sci. Agron., 25:35-44, 2003.

MITCHELL, J.K. Fundamentals of soils behavior. 2.ed. New York, Wiley, 1993. 437p.

MORAES, S.O. Heterogeneidade hidráulica de uma terra roxa estruturada. Piracicaba, Escola Superior de Agricultura "Luiz de Queiroz", 1991. 141p. (Tese de Doutorado)
NIELSEN, D.R. \& BIGGAR, J.W. Miscible displacement in soils: I. Experimental information. Soil Sci. Soc. Am. Proc., 25:1-5, 1961.

PASEKA, A.I.; IQBAL, M.Z. \& WALTERS, J.C. Comparison of numerical simulation of solute transport with observed experimental data in a silt loam subsoil. Environ. Geol., 9:977-989, 2000.

PUPISKY, H. \& SHAINBERG, I. Salt effects on the hydraulic conductivity of a sandy soil. Soil Sci. Soc. Am. J., 43:429433, 1979.

RADCLIFFE, D.E. \& RASMUSSEN, T.C. Soil water movement. In: SUMMER, M.E., ed. Handbook of soil science. Boca Raton, CRC Press, 2000. p.A879-A127.

REICHARDT, K. \& TIMM, L.C. Solo, planta e atmosfera: Conceitos, processos e aplicações. Barueri, Manole, 2004. 478p.

SHUKLA, M.K.; ELLSWORTH, T.R.; HUDSON, R.J. \& NIELSEN, D.R. Effect of water flux on solute velocity and dispersion. Soil Sci. Soc. Am. J., 67, 449-457, 2003.

STARRETT, S.K.; CHRISTIANS, N.E. \& AUSTIN, T.A. Comparing dispersivities and soil chloride concentrations of turfgrass-covered undisturbed and disturbed soil columns. J. Hydrol., 180:21-29, 1996.

YOUNGS, E.G. Hydraulic conductivity of saturated soils. In: SMITH, K.A. \& MULLINS, C.E., eds. Soil analysis: Physical methods. New York, M. Dekker, 1991. p.161-207. 